\title{
MULTIPHASE MODELING OF WAVE PROPAGATION OVER SEMICIRCULAR OBSTACLES USING WENO AND LEVEL SET METHODS
}

\author{
Tamer Kasem ${ }^{1}$ and Jun Sasaki ${ }^{1}$
}

\begin{abstract}
Wave propagation over a semicircular obstacle is studied. This problem is related to the design of semicircular breakwaters. These breakwaters are expected to have enhanced stability and were constructed in various places in China. Enhanced numerical modeling is done taking viscosity into account. The fifth order space accurate weighted essentially non-oscillatory (WENO) method is used to discretize the convection terms. As a result accurate results are obtained using simple options (uniform Cartesian grid, level set method). Wave generation is done using a numerical piston wave maker that is analogous to the real experiment. The model results are compared with free surface visualization and pressure measurements. Various features of the problem including wave drag and the flow field are revealed.
\end{abstract}

Keywords: Semicircular breakwater; Numerical model; WENO; Visualization

\section{INTRODUCTION}

Detached or nearshore breakwaters have been used extensively for coast protection or the creation of crescentic beaches (Reeve et al. 2004). One relatively new type of detached breakwaters is a semicircular breakwater. Breakwaters of this type which were first developed in Japan in the 1990s are expected to exhibit various advantages including enhanced sliding stability (Sasajima et al. 1994). Yuan and Tao (2003) reported that semicircular breakwaters were constructed in different places in China. In the present paper wave propagation over a semicircular obstacle is studied. This problem is directly related to the design of semicircular breakwaters. The study of Yuan and Tao (2003) is one of the few numerical studies where the present topic was considered. They used a potential flow solver neglecting viscosity. On the other hand Zhuang and Lee (1996) verified that a potential model yields large errors for separated flow behind a rectangular obstacle. Consequently, studying wave flow over a semicircular obstacle based on the potential flow assumption may lead to inaccurate results. The goal of the present work is to provide enhanced numerical modeling results of wave propagation over a semicircular obstacle taking viscosity into account.

In the present model WENO $5^{\text {th }}$ order method is used to discretize the momentum terms and to track the free surface motion. Two main advantages of this choice exist. The first advantage is that complex flow can be modeled using an equally spaced grid. This is due to the high resolution property of WENO method. It should be noted that other models relying on low order methods, adopt nonuniform grids placing finer grids where high resolution is needed. Three widespread models that adopt non-uniform grids are; "COBRAS" (Lin and Liu 1998), "VOFbreak" (Troch and De-Rouk 1998, 1999), and "CADMAS-SURF" (Interim Development Committee of a Numerical Wave Flume for Maritime Structure Design, 2001 and Isobe et al. 1999). Compared to a uniform grid, adopting a nonuniform grid consumes more effort. In addition determination of places where extra resolution is needed is a difficult task when strong free surface deformation and flow vortices occur simultaneously.

The second advantage of using WENO is related to tracking the free surface. In COBRAS, VOFbreak $^{2}$ and CAMAS-SURF the free surface is tracked using VOF method. A crucial step in VOF is free surface reconstruction (Scardovelli and Zaleski 1999). This step is not simple (Sethian and Smereka 2003). In the current study free surface is tracked using level set method. Reconstruction is avoided in the level set method, and the free surface is tracked using WENO.

These two advantages of WENO substantially reduce the effort required to develop the numerical model. The purpose of the current work is to provide accurate numerical results for the problem of wave propagation over a semicircular obstacle using our new simple model. In order to further clarify our motivation, the basic features of the models are explained briefly. Free surface visualization is done to validate the numerical results. The flow features are illustrated to provide enhanced understanding of the problem.

\section{NUMERICAL MODEL}

In this section a brief description of the numerical model adopted in this work is presented. A detailed description of the model including validation against various test cases is provided in Tamer and Sasaki (2010).

\footnotetext{
${ }^{1}$ Department of Civil Engineering, Yokohama National University, Tokiwadai, Hodogaya, Yokohama 240-8501, Japan
} 


\section{Governing Equations and Algorithm}

Neglecting surface tension, for a two-phase gas-liquid flow the incompressible Navier Stokes equations can be written in dimensionless form as (Sussman et al. 1994):

$$
\begin{gathered}
\frac{\partial u_{j}}{\partial x_{j}}=0 \\
\frac{\partial u_{i}}{\partial t}=-\frac{\partial\left(u_{i} u_{j}\right)}{\partial x_{j}}+\frac{1}{\rho R_{e}}\left[\frac{\partial\left\{\mu\left(\frac{\partial u_{i}}{\partial x_{j}}+\frac{\partial u_{j}}{\partial x_{i}}\right)\right\}}{\partial x_{j}}\right]-\frac{1}{\rho} \frac{\partial P}{\partial x_{i}}-\frac{L_{\infty}}{U_{\infty}{ }^{2}} g_{i}
\end{gathered}
$$

Eq. 1 and Eq. 2 are the continuity and momentum equations, respectively, where $u_{i}$ is the dimensionless velocity in the $i$ direction. $g_{i}$ is the gravity force defined using Kronecker delta as $g_{i}=-9.8 \delta_{i 2}$. The index $i=1,2$ stands for the horizontal and vertical $x, y$ directions, respectively; $L_{\infty}$ and $U_{\infty}$ are the reference length and velocity scales, respectively; $R_{e}$ is the dimensionless Reynolds number defined as $R_{e}=\rho_{\infty} U_{\infty} L_{\infty} / \mu_{\infty} ; P, \rho$ and $\mu$ are are the dimensionless pressure, density and viscosity that are normalized using $\rho_{\infty} U_{\infty}{ }^{2}, \rho_{\infty}$ and $\mu_{\infty}$, respectively.

In the present model a methodology that is similar to the MAC method is adopted. A staggered grid is used. Viscous and convective terms on the right hand side of Eq. 2 are discretized using variables at the current time step. The divergence free constraint (Eq. 1) is fulfilled, and the pressure is computed by solving a Poisson equation for pressure. Finally, the velocity components at the new time step are evaluated.

Multiphase flow is modeled using the level set method, where liquid and gas regions are identified using a color function termed as $\phi$. This function is assigned to the distance from the gas-liquid interface. The time variation of $\phi$ is governed by:

$$
\frac{\partial \phi}{\partial t}+\frac{\partial\left(\phi u_{i}\right)}{\partial x_{i}}=0
$$

Fluid properties are calculated using Eq. 4:

$$
\begin{gathered}
\rho=\rho_{L} \phi_{c o m p}+\rho_{G}\left(1-\phi_{c o m p}\right) \\
\mu=\mu_{L} \phi_{c o m p}+\mu_{G}\left(1-\phi_{c o m p}\right)
\end{gathered}
$$

where $\mu_{L}, \rho_{L}, \mu_{G}$ and $\rho_{G}$ are the dynamic viscosities and densities of the liquid and gas, respectively. $\phi_{\text {comp }}$ is computed using Eq. 5.

$$
\phi_{\text {comp }}=\left\{\begin{array}{cc}
0 & \text { if } \phi<-\delta \\
1 & \text { if } \phi>\delta \\
0.5\left(1+\sin \left(\frac{\pi \phi}{2 \delta}\right)\right) & \text { else }
\end{array}\right.
$$

Here, $\delta$ which represents the liquid-gas interface thickness is defined using the grid size $\Delta$ as $\delta=1.5 \Delta$. An important step to be done after solving Eq. 3 using WENO is Re-initialization. This step assures that the slope of $\phi$ is kept equal to unity. For more details about the re-initialization the reader is referred to Tamer and Sasaki (2010) and the references cited there.

\section{WENO Discretization Method}

The convection terms in Eq. 2 and Eq. 3 are discretized using WENO method. Consequently WENO plays an important role in the current numerical model. The main features and motivations for using WENO will be presented briefly in this section. For a detailed explanation the reader is referred to Shu (1998). In WENO method a concept that it is similar to finite difference methods is adopted. These methods are based on interpolation using polynomials or other functions. In the approximation theory, it is well known that the wider the stencil, the higher the order of accuracy. However interpolation using second or higher order accuracy leads to spurious oscillations near a discontinuity (Gibbs phenomena). These oscillations often lead to numerical instability. The concept adopted in 
WENO to avoid this problem can be explained if we consider the discretization of the horizontal advection term $\partial(u f) / \partial x$ at point $i$ :

$$
\left.\frac{\partial(u f)}{\partial x}\right|_{i}=\frac{\widehat{u f}_{i+1 / 2}-\widehat{u f}_{i-1 / 2}}{\Delta x}+O\left(\Delta x^{5}\right)
$$

In Eq. $6, u$ is the velocity component in $x$ direction, $\Delta x$ is the grid size, and $f$ can be any scalar quantity (for example $\phi$ in Eq. 3). $\widehat{u f}_{i+1 / 2}$ and $\widehat{u f}_{i-1 / 2}$ are defined as the fluxes at the right and left boundaries of cell $i$. In order to achieve fifth order accuracy $\widehat{u f}_{i-1 / 2}$ is calculated using five points; $[i-2, i+2]$ for upwind approximation, or $[i-3, i+1]$ for downwind approximation (Fig. 1).

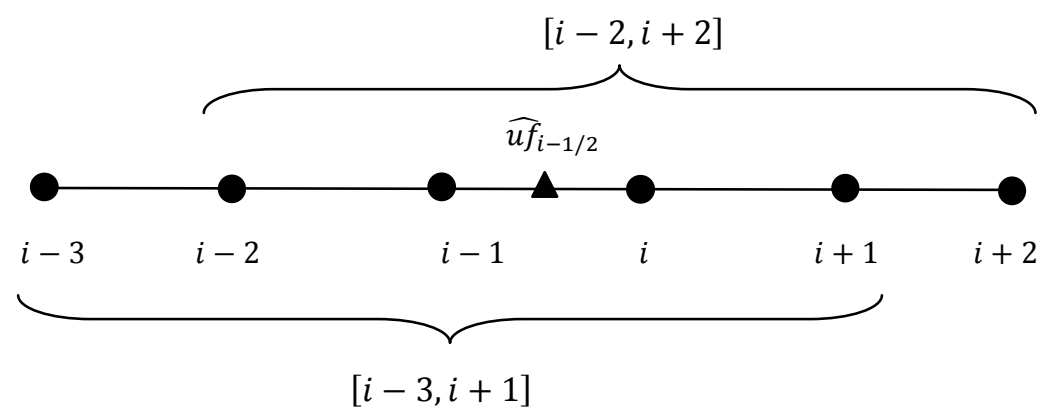

Figure 1. Downwind and upwind stencils for the flux at $i-1 / 2$.

The choice between both approximations is done based on the sign of $u$ to ensure that the flow information is obtained from the correct direction. For both choices $\widehat{u f}_{i+1 / 2}$ is calculated from a weighted sum of the flux calculated from three sub-stencils. For instance the downwind approximation of $\widehat{u f}_{i+1 / 2}$ includes the three points sub-stencils $[i-3, i-1],[i-2, i]$ and $[i-1, i+1]$. Finally the flux is obtained as the following weighted sum:

$$
\widehat{u f}_{i-1 / 2}=\widetilde{\omega}_{1} \overline{u f}_{1}+\widetilde{\omega}_{2} \overline{u f}_{2}+\widetilde{\omega}_{3} \overline{u f}_{3}
$$

In Eq. 7, $\overline{u f}_{1}, \overline{u f}_{2}$ and $\overline{u f}_{3}$ are estimates for the flux obtained from each of the sub-stencils already mentioned. $\widetilde{\omega}_{1}, \widetilde{\omega}_{2}$ and $\widetilde{\omega}_{3}$ are weights assigned to each sub-stencil. In order to obtain an oscillation free approximation, the weights are adjusted to yield high values for smooth sub-stencils and vice versa.

Once the fluxes are calculated at the cell boundaries they are substituted in Eq. 6 to evaluate the convection derivative. The same procedure is adopted to calculate the vertical derivative $\partial(u f) / \partial y$. For a Cartesian grid, the calculation steps of the vertical and horizontal derivatives are totally independent.

To reveal the advantages of WENO method, Eq. 8 is solved, subject to the initial condition given by Eq. 9 .

$$
\begin{gathered}
\frac{\partial f}{\partial t}+C \frac{\partial f}{\partial x}=0 \\
f(x, t=0)=b(x)=\left\{\begin{array}{cc}
1 & 1<x<2 \\
0 & \text { else }
\end{array}\right.
\end{gathered}
$$

Here $C$ is a constant equal to 10.0. Using characteristics it can be shown that the exact solution of this problem is translating the initial condition (square wave) to the right with the constant velocity $C$. The numerical domain extends between $0<x<10$. The simulation is done for a maximum time of 0.7, adopting 160 mesh points and 900 time steps. In Fig. 2 the exact and the numerical solutions at the maximum time are shown. The numerical result of CIP method (Yabe et al. 1991) is shown to illustrate the advantage of WENO5 method. As expected the profile is translated to a total distance equal to 7. The CIP method yields spurious oscillations near the discontinuity. On the other hand WENO5 method yields a solution that is more sharp and oscillation-free. 


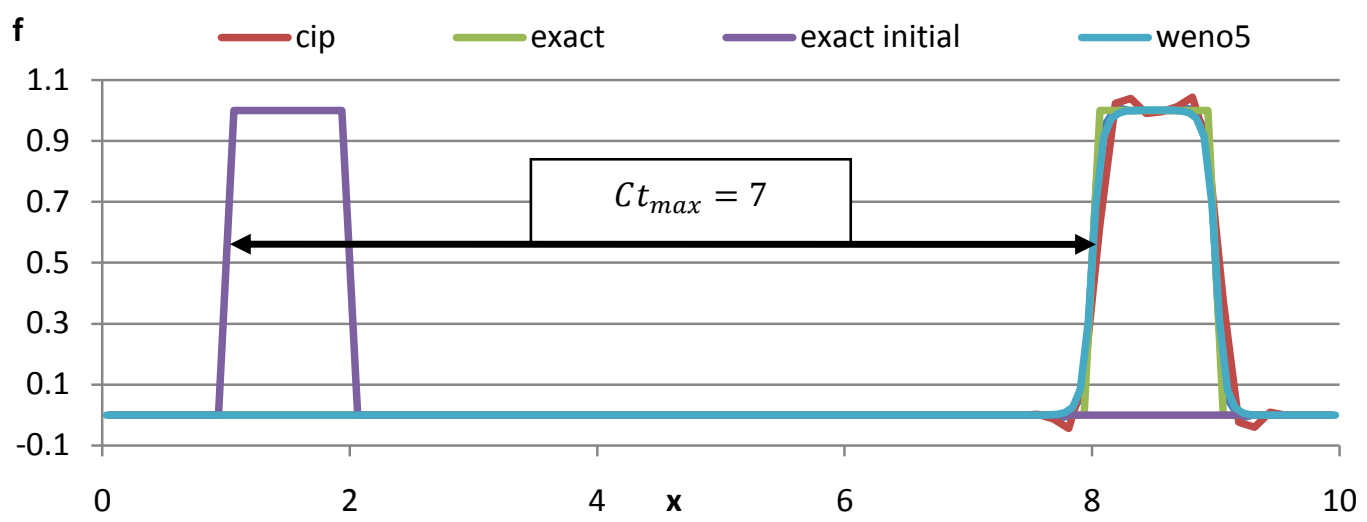

Figure 2. Initial profile and final profiles of $f$ at $t=0.7$.

\section{Problem Setup and Wave Generation}

The problem of wave propagation over a semicircular obstacle is sketched in Fig. 3. The reference density and viscosity are defined as $\rho_{\infty}=\rho_{w}$ and $\mu_{\infty}=\mu_{w}$ using water values. Also length, velocity and time scales are defined in terms of the water depth as $L_{\infty}=d, U_{\infty}=\sqrt{g d}$ and $t_{\infty}=\sqrt{d / g}$. In Table 1, the definitions of the dimensionless parameters that govern the problem along with the values corresponding to the experimentally visualized case are shown. The dimensionless wave length and period are related by the dispersion relation (Dean and Dalrymple 1984):

$$
\frac{2 \pi}{\bar{T}^{2}}=\frac{\tanh (2 \pi / \bar{L})}{\bar{L}}
$$

Waves are generated numerically by incorporating a piston wave maker in the domain. The dimensionless wave maker displacement $\bar{\xi}$ is determined as (Dong and Huang 2004):

$$
\begin{gathered}
\bar{\xi}=-\bar{\xi}_{0}\left[\cos (\sigma t)+\frac{\bar{H}}{4 n_{1}}\left(\frac{0.75}{\sinh (k)^{2}}-\frac{n_{1}}{2}\right) \sin (2 \sigma t)\right] \\
\bar{\xi}_{0}=\frac{\bar{H} \times n_{1}}{2 \tanh (k)} \\
n_{1}=\frac{1}{2}\left(1+\frac{2 k}{\sinh 2 k}\right)
\end{gathered}
$$

Here $k=2 \pi / \bar{L}$ and $\sigma=2 \pi / \bar{T}$ are the dimensionless wave number and frequency, respectively. The second order term in Eq. 11 is added to eliminate second-order free waves. Such undesired free waves may result if the piston motion is governed by the first order formula: $\bar{\xi}=-\bar{\xi}_{0} \cos (\sigma t)$. The generated waves are Stokes second order waves of permanent form. The piston velocity is calculated from the time derivative of Eq. 11. This value is assigned directly to the velocity in the cells that reside in the piston. This method of wave generation has the following advantages:

1. The method is directly equivalent to the real wave maker. Hence the extensive literature related to wave maker theory can be applied in a straightforward manner.

2. Since all the outer boundaries are stationary walls (zero normal velocity), the velocity and pressure boundary conditions can be applied using the ordinary formulas in text books (see for example Anderson et al. 1984). It should be noted that a detailed literature exists regarding applying the correct pressure boundary conditions for non-zero normal velocity (including Gresho and Sani 1987, Gresho 1991 and Sani et al. 2006). Hence avoiding this type of conditions reduces the modeling effort.

3. Another method of wave generation that is adopted in COBRAS model is to include an artificial source of mass in the domain (Lin and Liu 1999). Special care should be allocated to adjust the 
size, location and the strength of this source. On the other hand, the method adopted in the current paper does not include any artificial parameters to be adjusted.

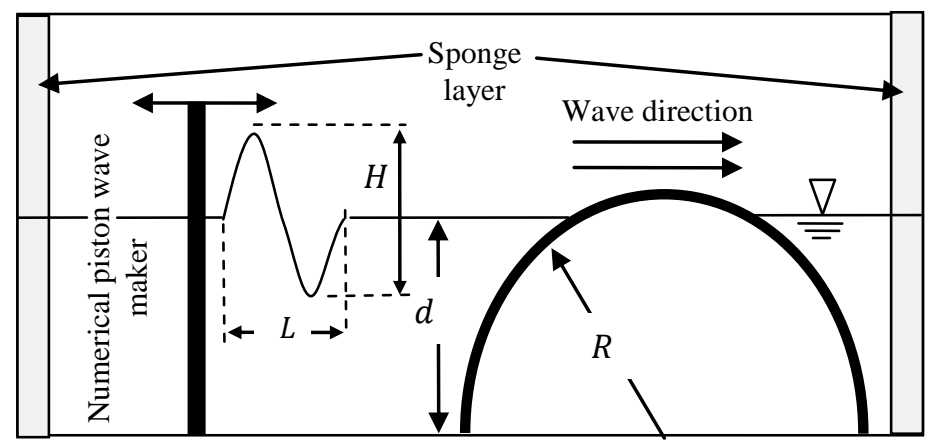

Figure 3. Definition of the problem of wave propagation over a semicircular obstacle (drawing not to scale).

\section{VISUALIZATION}

Experimental visualization of the wave propagation over a semicircular obstacle is provided. The $17.3 \mathrm{~m}$ length wave flume in Yokohama National University was used. Waves were generated using a piston type wave maker. The videos were taken using SANYO VPC-HD700 digital video camera (640×480 pixels, 30fps). Visualization results are provided for the case listed in Table 1. A regular wave was generated with a period $T=1 \mathrm{~s}$. A transparent grid of $1 \mathrm{~cm} \times 1 \mathrm{~cm}$ cell size was used to provide adequate visualization. Illumination was enhanced using two halogen lamps each of $500 \mathrm{~W}$ power. The images resulting from experimental visualization covered an area of $33 \mathrm{~cm}$ width, extending from $4 \mathrm{~cm}$ to $26 \mathrm{~cm}$ heights.

\begin{tabular}{|c|c|c|c|c|c|c|}
\hline $\begin{array}{c}\text { Dimensionless } \\
\text { wave height } \\
\bar{H}=H / d\end{array}$ & $\begin{array}{c}\begin{array}{c}\text { Reynolds } \\
\text { number }\end{array} \\
R_{e} \\
=\frac{\sqrt{g} d^{1.5} \rho_{w}}{\mu_{w}}\end{array}$ & $\begin{array}{c}\text { Air- } \\
\text { water } \\
\text { density } \\
\text { ratio } \\
\frac{\rho_{a}}{\rho_{w}}\end{array}$ & $\begin{array}{c}\text { Air- } \\
\text { water } \\
\text { viscosity } \\
\text { ratio } \\
\frac{\mu_{a}}{\mu_{w}}\end{array}$ & $\begin{array}{c}\text { Dimensionless } \\
\text { obstacle Radius } \\
\quad \bar{R}=R / d\end{array}$ & $\begin{array}{l}\text { Dimensionless } \\
\text { wave period } \\
\bar{T}=T \sqrt{g / d}\end{array}$ & $\begin{array}{c}\text { Dimensionless } \\
\text { wave length } \\
\bar{L}=L / d\end{array}$ \\
\hline 0.4 & $2.03 \times 10^{5}$ & $\begin{array}{l}1.2 \\
\times 10^{-3} \\
\end{array}$ & $\begin{array}{l}1.97 \\
\times 10^{-2} \\
\end{array}$ & 1.15 & 7.754 & 6.902 \\
\hline
\end{tabular}

\section{RESULTS}

\section{Semicircular Obstacle}

In the current section the numerical results of the current model, applied to the problem of wave propagation over a semicircular obstacle are shown. The results correspond to the visualized case whose parameters are shown in Table 1 . For the numerical simulation the following parameters are chosen; left and right sponge layer widths $3 d$; maximum simulation time of $9 \bar{T} ; 36,000$ time steps; domain width and height of $38 d$ and $2.2 d$, respectively; numerical grid of $760 \times 96$ cells in horizontal and vertical directions, respectively; semicircle center position at $30 \mathrm{~d}$; piston wave maker position at $5 d$; and finally piston thickness of 2 horizontal numerical cells and height $1.8 d$.

The streamlines at air and water near the wave maker are shown for one wave period at five instants in Fig. 4. Air motion is characterized by two vortices on both sides of the wave maker. The size and the intensity of each vortex vary periodically with time in response to the oscillatory motion of the wave maker. 



Figure 4. Streamlines for air and water near the wave maker at $2.5 \mathrm{~s}, 2.7 \mathrm{~s}, 2.9 \mathrm{~s}, 3.1 \mathrm{~s}$ and $3.3 \mathrm{~s}$, from top to bottom, respectively.

In Fig. 5 both experimental visualization results and calculations are shown. Visualization is provided at five instants with $0.2 \mathrm{~s}$ separation between successive frames. While experimental results reveal only the free surface location, both the free surface and stream lines are provided in the numerical simulation results. A close agreement exists between numerical and experimental free surface locations. It should be noted that strong free surface deformation and flow vortices occur simultaneously. This reveals the capability of WENO5 high order method of capturing complex flow features using a uniform grid. 



Figure 5. Experimental visualization (right) and calculated free surface and stream lines (left) are shown from up to down at $5.65 \mathrm{~s}, 5.85 \mathrm{~s}, 6.05 \mathrm{~s}, 6.25 \mathrm{~s}$ and $6.45 \mathrm{~s}$, respectively. 


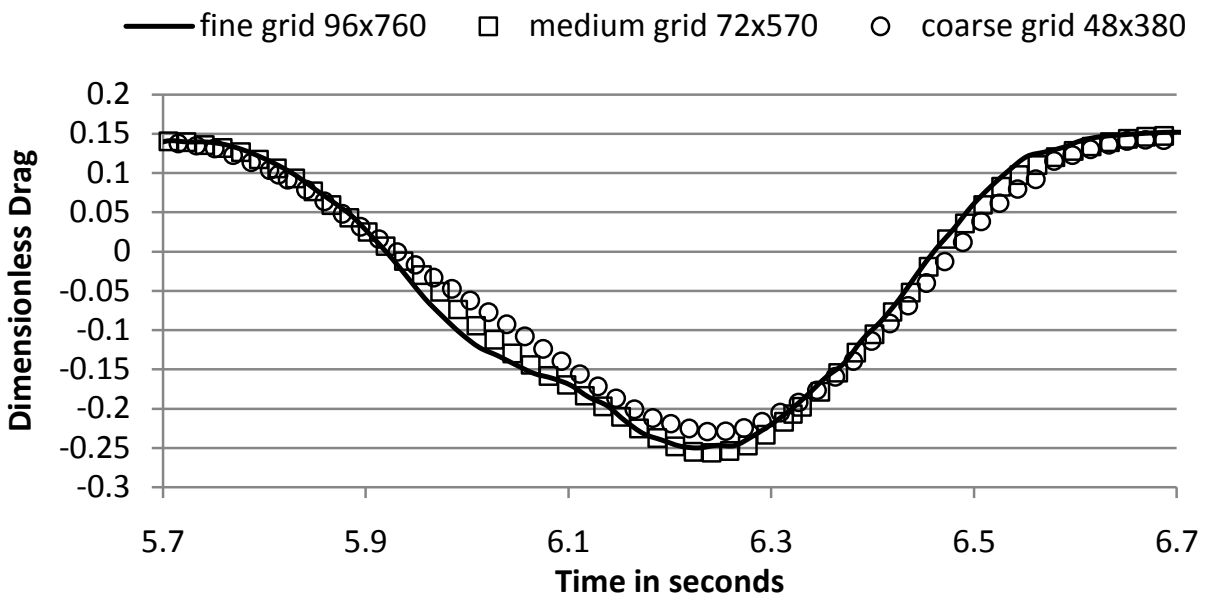

Figure 6. Temporal variation of the dimensionless horizontal drag during one wave period for different grids.

In Fig. 6 the horizontal dimensionless drag force during one period is shown for three grids. The dimensionless drag is defined as

$$
\bar{D}=\frac{1}{d \rho_{w} U_{\infty}^{2}} \oint P d y
$$

Since the results of the medium and the fine grids are very close, it is confirmed that converged drag values are computed. The magnitude of the minimum drag force in anti-wave direction is considerably higher than the magnitude of the maximum drag in the wave direction. Flow visualization at an instant close to which the drag reaches its minimum value, is provided in Fig. 5 at $6.25 \mathrm{~s}$. Flow separation and considerable water level drop on the obstacle upstream side can be observed. It should be noted that this instant corresponds roughly to the wave trough occurrence time.

Goda (1985) provided detailed results for the problem of wave contact to a vertical section. These results can be summarized as: under the trough the wave pressure becomes less than the hydrostatic pressure under the still water level; as a result the net force is directed towards the offshore; the wave pressure under the trough may become larger than that under the crest; under such a condition an upright section may slide toward the offshore during heavy wave action. An estimate for the maximum wave force in anti wave direction affecting a vertical wall for the current case is 0.21 (Goda 1985). This value is close to the current model results which is 0.25 (Fig. 6). Consistency between the results of the current work and those related to the vertical wall is clear.

\section{Semicircular Breakwater}

In the current Subsection the current model is used to simulate wave flow over a semicircular breakwater. Unlike the simplified case already studied, the detailed geometry of the structure is considered. In Fig. 7 the problem geometry and setup is illustrated. The values of the problem geometrical and physical parameters are given in Table 2. Those values correspond to the test case given by Yuan and Tao (2003), identified as: cross section number 5 with water level at breakwater top.

Yuan and Tao (2003) provided data on pressure measurements performed using a large scale model with $R=7.2 \mathrm{~m}$ and $d_{1}=8 \mathrm{~m}$. These measurements are rather valuable since access to similar large scale test facilities is generally limited.

The incoming wave height value reported by Yuan and Tao (2003) is $H / d_{1}=0.59$. This value is very close to the maximum possible wave height (see Massel (1996) page 301) and is much bigger than the limit of second order wave theory. To generate this high amplitude wave a lower amplitude wave is generated $(H / d=0.45)$ in a relatively deep region, then the wave is shoaled to a shallow region to increase its amplitude. This is the aim of introducing the slope of height $h_{s l p}$ in the domain. This mechanism is expected to yield reliable results (see Hughes (1993) page 379). 


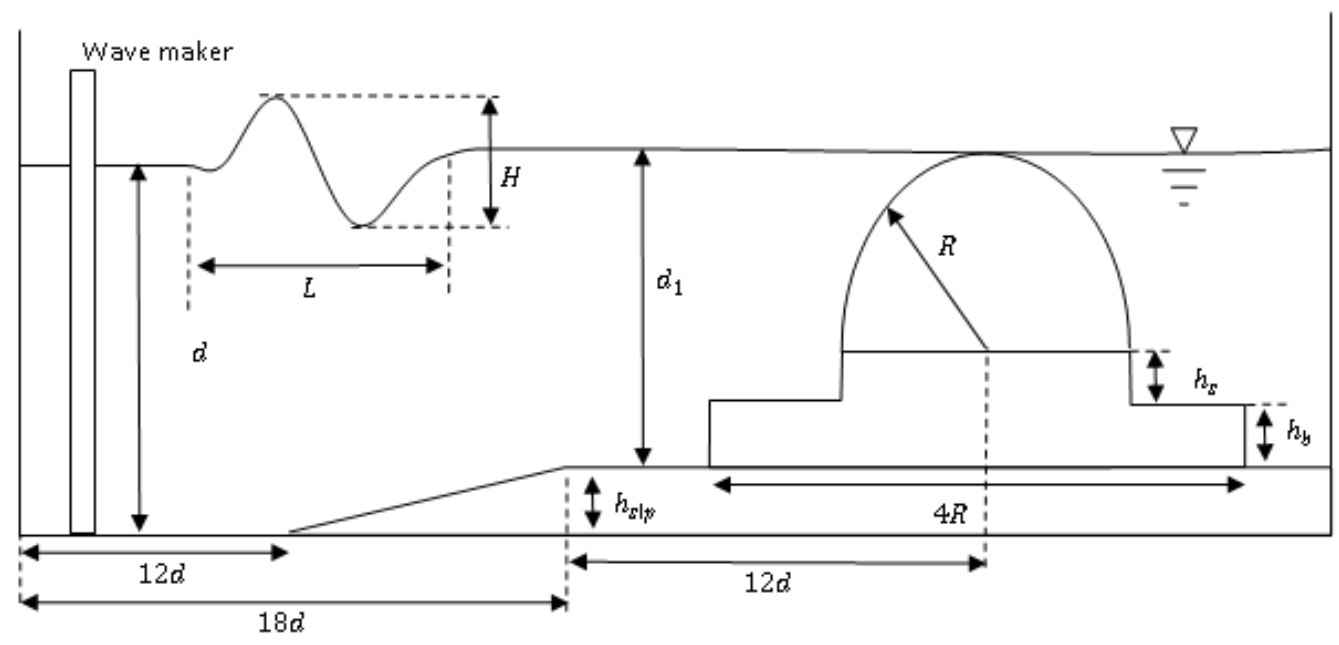

Figure 7. Problem setup and semicircular breakwater geometry.

\begin{tabular}{|c|c|c|c|c|c|c|c|}
\hline \multicolumn{7}{|c|}{ Table 2. Values of physical and geometrical parameters. } \\
\hline $\bar{H}=H / d$ & $R_{e}=\frac{\sqrt{g} d^{1.5} \rho_{w}}{\mu_{w}}$ & $\bar{R}=R / d$ & $\bar{T}=T \sqrt{g} / d$ & $\bar{L}=L / d$ & $h_{s} / d$ & $h_{b} / d$ & $h_{s l p} / d$ \\
\hline 0.45 & $1.083 \times 10^{8}$ & 0.68 & 6.53 & 5.525 & 0.029 & 0.048 & 0.24 \\
\hline
\end{tabular}

For the numerical simulation the following parameters are chosen; domain height $1.8 d$; numerical grid of $760 \times 88$ cells in horizontal and vertical directions, respectively; and piston wave maker height $1.5 \mathrm{~d}$. The rest of the numerical parameters are similar to those mentioned in the previous Subsection.

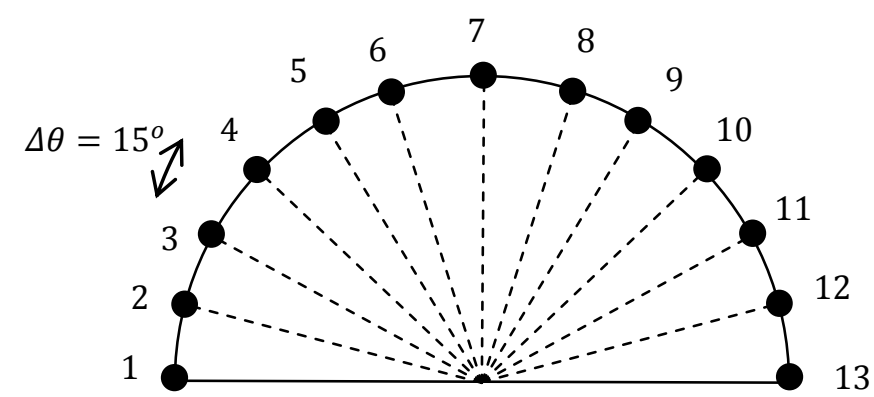

Figure 8. Locations where pressure measurements are provided.

a experiment $\quad-$ potential model $\quad$ Current model



Figure 9. Results for pressure at measurement locations. 

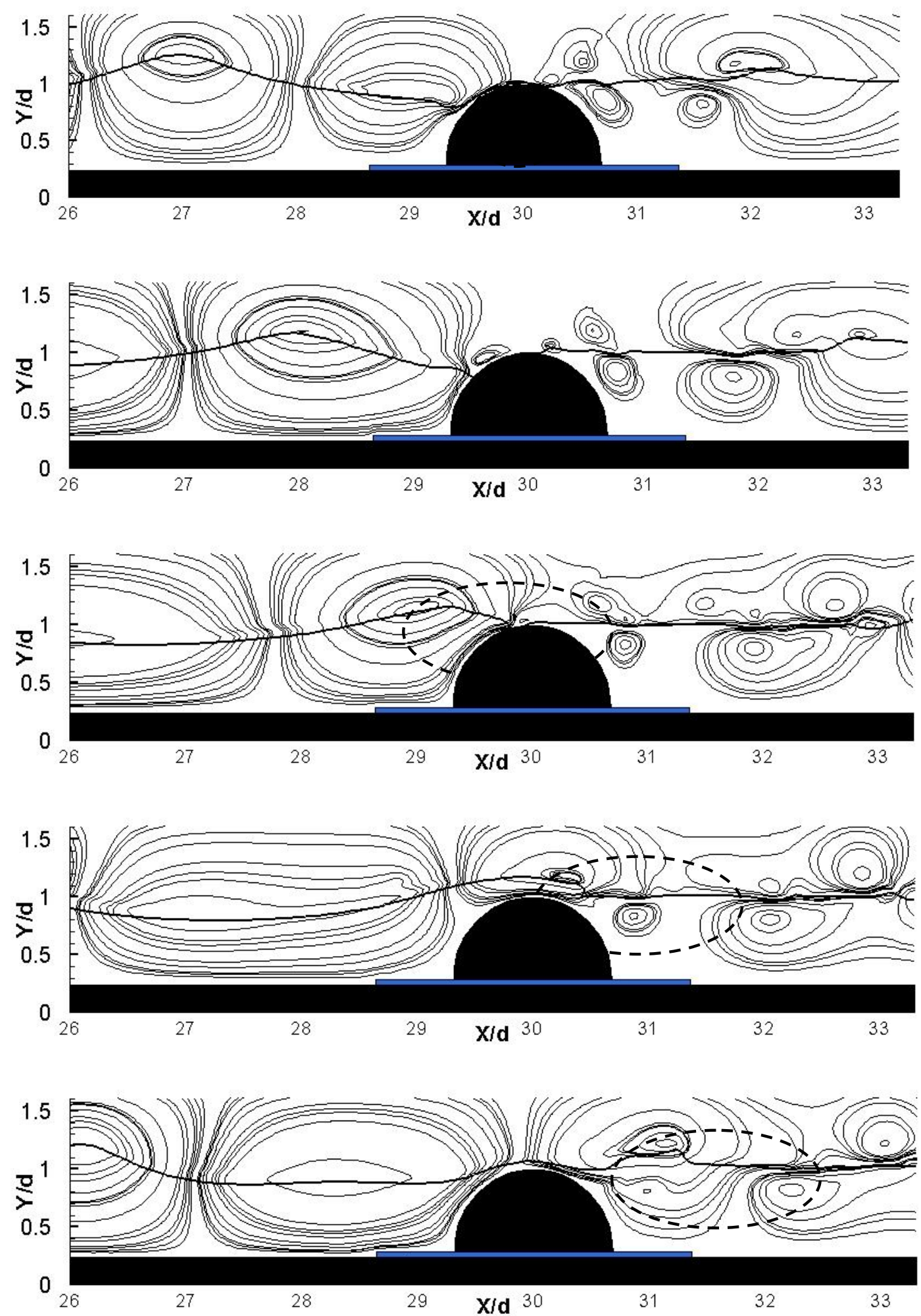

Figure 10. Streamlines and free surface near the breakwater at five instants in one wave period.

In Fig. 9 the wave pressure at the locations defined in Fig. 8 is shown at the instant of minimum wave force (maximum wave force in anti wave direction). The wave pressure is evaluated by subtracting the hydrostatic pressure from the absolute pressure. The current model results are shown 
along with the measured values and the calculations of the potential model of Yuan and Tao (2003). The results of the current model are closer to the experimental values. This is expected since for the current case separation vortices should occur near the breakwater, consequently the potential flow assumption is invalid.

In Fig. 9 streamlines and free surface are shown near the semicircular breakwater at $t \sqrt{d / g} / T=$ 6.4, 6.6, 6.8, 7.0 and 7.2. Wave steepening as the crest approaches the obstacle can be observed at the third instant $t \sqrt{d / g} / T=6.8$ indicated by the dashed oval. A forward breaking jet which occurs after the crest crosses the obstacle can be identified at $t \sqrt{d / g} / T=7.0$ and 7.2. The drop in the water level in the upstream side can be obserevd at the beginning of the wave period at $t \sqrt{d / g} / T=6.4$.

\section{CONCLUSIONS}

In this paper the problem of wave propagation over a semicircular obstacle was studied using a new simple model. This model adopts WENO method to discretize the flow equations and to capture the free surface motion. The following conclusions should be pointed:

1. The agreement between the experimental visualization results and pressure measurements with the results of our model, proves the validity of the current work.

2. The numerical results revealed the existence of flow vortices in the air and the water. Also, the results of our model are closer to the pressure measurements than those of the potential model. Consequently the importance of including the flow viscosity is verified.

3. The method adopted for wave generation in the current work proved to be reliable.

4. An important finding to be stressed is the high wave force affecting the semicircular obstacle in the anti-wave direction. This finding should provide important guidance for future design.

The results of the proposed model with a relatively simple formulation are very promising. Further modification of this model for more complex situations is planned. These situations include modeling random waves, and permeable perforated walls in the semicircular structure.

\section{ACKNOWLEDGMENTS}

The present study is partially funded by JSPS Grant in Aid for Scientific Research (B) No. 15360263.

\section{REFERENCES}

Anderson, D, J Tannehill, and R Pletcher. 1984. Computational Fluid Mechanics and Heat Transfer, Taylor \& Francis, 816pp.

Dean, R., and R. Dalrymple. 1984. Water Wave Mechanics for Engineers and Scientists, Prentice-Hall, $353 \mathrm{pp}$.

Dong, C., and C. Huang. 2004. Generation and propagation of water waves in a two-dimensional numerical viscous wave flume, Journal of Waterway, Port, Coastal \& Ocean Engineering, 130, 143-153.

Goda, Y. 1985. Random Seas and Design of Maritime Structures, University of Tokyo Press, 323 pp.

Gresho, P. 1991. Incompressible Fluid Dynamics: Some Fundamental Formulation Issues, Annual Review of Fluid Mechanics, 23, 413-453.

Gresho, P., and R. Sani. 1987. On pressure boundary conditions for the incompressible Navier-Stokes equations, International Journal for Numerical Methods in Fluids, 7, 1111-1145.

Hughes, S. 1993. Physical Models and laboratory Techniques in Coastal Engineering, World Scientific, $550 \mathrm{pp}$.

Interim Development Committee of a Numerical Wave Flume for Maritime Structure Design. 2001. Numerical Wave Flume Theory and Development, Coastal Development Institute of Technology Japan, (in Japanese).

Isobe, M., S. Takahashi, , S. Yu, T. Sakakiyama, , K. Fujima, K. Kawasaki, , Q. Jiang, M. Akiyama, and H. Oyama. 1999. Interim development of a numerical wave flume for maritime structure design, Proceedings of Civil Engineering in the Ocean, JSCE, 321-326, (in Japanese).

Lin, P., and P. Liu. 1998. A numerical study of breaking waves in the surf zone, Journal of Fluid Mechanics, 359, 239-264.

Lin, P., and P. Liu. 1999. Internal Wave-Maker for Navier-Stokes Equations Models, Journal of Waterway, Port, Coastal \& Ocean Engineering, 125, 207-215.

Massel, R. 1996. Ocean Surface Waves: Their Physics and Prediction, World Scientific, 491 pp. 
Reeve, D., A. Chadwick, and C. Fleming. 2004. Coastal Engineering, Processes, Theory and Design Practice, Spon Press, 416 pp.

Sani, R., J. Shen, O. Pironneau, and P. Gresho. 2006. Pressure boundary condition for the timedependent incompressible Navier-Stokes equations, International Journal for Numerical Methods in Fluids, 50, 673-682.

Sasajima, H., T. Koizuka, H. Sasayama, Y. Niidome, and T. Fujimoto. 1994. Field demonstration test on a semi-circular breakwater, Proceedings of the International Conference on Hydro-Technical Engineering for Port \& Harbor Construction, HYDRO-PORT'94, Yokosuka, Japan, 593-615.

Scardovelli, R., and S. Zaleski. 1999. Direct numerical simulation of free-surface and interfacial flow, Annual Review of Fluid Mechanics, 31, 567-603.

Sethian, J., and P. Smereka. 2003. Level set methods for fluid interfaces, Annual Review of Fluid Mechanics, 35, 341-372.

Shu, C. 1998. Essentially non-oscillatory and weighted essentially non-oscillatory schemes for hyperbolic conservation laws, Lecture Notes in Mathematics, Vol. 1697, in Advanced Numerical Approximation of Nonlinear Hyperbolic Equations, edited by A Quarteroni, 325-432.

Sussman, M., P. Smereka, and S. Osher. 1994. A level set approach for computing solutions to incompressible two-phase flow, Journal of Computational Physics, 114, 146-159.

Tamer, K., and J. Sasaki. 2010. Multiphase modeling of wave propagation over submerged obstacles using weno and level set methods, Coastal Engineering Journal, (in press).

Troch, P., and J. De-Rouk. 1998. Development of two-dimensional numerical wave flume for wave interaction with rubble mound breakwaters, Proceedings of the 26th International Conference on Coastal Engineering, ASCE, 1638-1649.

Troch, P., and J. De-Rouk. 1999. An active wave generating-absorbing boundary condition for VOF type numerical model, Coastal Engineering, 38, 223-247.

Yabe, T., T. Ishikawa, P. Wang, T. Aoki, Y. Kadota, and F. Ikeda. 1991. A universal solver for hyperbolic equations by cubic-polynomial interpolation II. Two- and three-dimensional solvers, Computer Physics Communications , 66, 233-242.

Yuan, D., and J. Tao. 2003. Wave forces on submerged, alternately submerged, and emerged semicircular breakwaters, Coastal Engineering , 48, 75-93.

Zhuang, F., and J. Lee. 1996. A Viscous Rotational Model for Wave Overtopping over Marine Structure, Proceedings of the 25th International Conference on Coastal Engineering, Orlando, Florida: ASCE, 2178-2191. 